\title{
Bonding of Gold Nanoclusters to Oxygen Vacancies on Rutile $\mathrm{TiO}_{2}(110)$
}

\author{
E. Wahlström, ${ }^{1}$ N. Lopez, ${ }^{2, *}$ R. Schaub,${ }^{1}$ P. Thostrup, ${ }^{1}$ A. Rønnau, ${ }^{1}$ C. Africh,${ }^{1, \dagger}$ E. Lægsgaard, ${ }^{1}$ \\ J. K. Nørskov, ${ }^{2}$ and F. Besenbacher ${ }^{1, \sharp}$ \\ ${ }^{1}$ CAMP, iNANO and Department of Physics and Astronomy, University of Aarhus, DK-8000 Aarhus C, Denmark \\ ${ }^{2}$ CAMP and Department of Physics, Technical University of Denmark, DK-2800 Lyngby, Denmark
} (Received 19 July 2002; published 14 January 2003)

Through an interplay between scanning tunneling microscopy (STM) and density functional theory (DFT) calculations, we show that bridging oxygen vacancies are the active nucleation sites for $\mathrm{Au}$ clusters on the rutile $\mathrm{TiO}_{2}(110)$ surface. We find that a direct correlation exists between a decrease in density of vacancies and the amount of Au deposited. From the DFT calculations we find that the oxygen vacancy is indeed the strongest Au binding site. We show both experimentally and theoretically that a single oxygen vacancy can bind $3 \mathrm{Au}$ atoms on average. In view of the presented results, a new growth model for the $\mathrm{TiO}_{2}(110)$ system involving vacancy-cluster complex diffusion is presented.

DOI: 10.1103/PhysRevLett.90.026101

PACS numbers: 68.47.Jn, 68.37.Ef

Titanium dioxide is one of the metal oxides that has attracted the most research interest over the past 30 years. Part of the interest stems from the ability of photoactivated titanium dioxide to split water [1,2]. Additional interest derives from the role of $\mathrm{TiO}_{2}$ as a support for metal catalysts, particularly since Haruta et al. [3] demonstrated that $\mathrm{Au}$ acts as a catalyst for low-temperature $\mathrm{CO}$ oxidation when dispersed as nanoclusters on a $\mathrm{TiO}_{2}$ support. It has been shown that the size of the Au clusters has a significant effect on the catalytic activity, with a peak in activity for $\sim 30 \AA$ wide Au clusters [4].

It is important to investigate the exact nature of the metal/oxide bond to understand not only the growth mechanism of metal clusters, but also to reveal the fundamental processes behind the catalytic activity of oxidesupported metal catalysts $[5,6]$. It has been speculated that surface defects may alter the electronic configuration of Au nanoparticles to enable catalytic reactions such as $\mathrm{CO}$ oxidation. Small Au clusters $\left(\mathrm{Au}_{8}\right)$ on another oxide support, $\mathrm{MgO}(100)$, were recently shown both experimentally and theoretically to be active for $\mathrm{CO}$ oxidation only if the Au clusters nucleate at oxygen vacancies [7].

In this Letter we present a detailed study of the interaction of $\mathrm{Au}$ nanoparticles with the most abundant surface defect on the (110) surface of reduced rutile $\mathrm{TiO}_{2}$ : bridging oxygen vacancies. Through an interplay between high-resolution scanning tunneling microscopy (STM) and density functional theory (DFT) we show that the nucleation and growth of $\mathrm{Au}$ clusters on the rutile $\mathrm{TiO}_{2}(110)$ surface is intimately related to the presence of surface oxygen vacancies and that larger Au clusters bind very weakly to the stoichiometric surface. By monitoring the temperature dependence of the cluster size distribution and the oxygen vacancy density we find that an oxygen vacancy accommodates 3-5 Au atoms on average. We show that medium-sized clusters (area $\sim 250 \AA^{2}$ ) are destabilized at room temperature (RT), providing evidence for mobility of Au-vacancy complexes. A new growth model for $\mathrm{Au} / \mathrm{TiO}_{2}(110)$ is presented.

The experimental setup and sample preparation are described elsewhere [8]. Au was deposited from an $e$-beam evaporator (Oxford Applied Research). The $\mathrm{Au}$ evaporation rate was quantitatively calibrated from STM measurements of $\mathrm{Au}$ deposited at $300 \mathrm{~K}$ on a $\mathrm{Ni}(111)$ surface (see, e.g., [9]) to be $0.01 \mathrm{ML} / \mathrm{min}$ [where one monolayer (ML) is defined as the packing density of an $\mathrm{Au}(111)$ plane, $1.39 \times 10^{15}$ atoms $\cdot \mathrm{cm}^{-2}$ ]. As is shown below, Au clusters bind very weakly to the stoichiometric surface. As a consequence, they can be dramatically influenced by the scanning process: at currents higher than $1 \mathrm{nA}$, a majority of Au clusters disappear from the scanning area. A new preamplifier has been developed to facilitate the lowest possible tunneling currents and high feedback gains. All presented STM images are acquired with $<0.1 \mathrm{nA}$ and $1.2 \mathrm{~V}$.

The theoretical modeling was performed within the DFT approach applied to periodic slabs containing three $\mathrm{O}-\mathrm{Ti}_{2} \mathrm{O}_{2}-\mathrm{O}$ units separated by $10 \AA$ of vacuum. $\mathrm{Au}$ is adsorbed on one side. For further details, see $[8,10]$.

Figure 1(a) shows an STM image of the clean surface prior to Au deposition. The STM image contrast on the $\mathrm{TiO}_{2}(110)$ surface has been shown to be dominated by electronic rather than geometric effects [11]: the protruding rows are assigned to fivefold coordinated $\mathrm{Ti}$ atoms, and the troughs are accordingly identified as bridging oxygen rows. In the present context, the most important feature is the bright spots observed between Ti rows, assigned to bridging oxygen vacancies [12]. In a recent paper [8], we demonstrated the ability of our STM to distinguish between bridging oxygen vacancies and bridging hydroxyl groups (both species displaying a similar signature in STM images), the latter originating from the dissociation of water molecules at oxygen vacancy sites. In the present study, special care has been taken to 

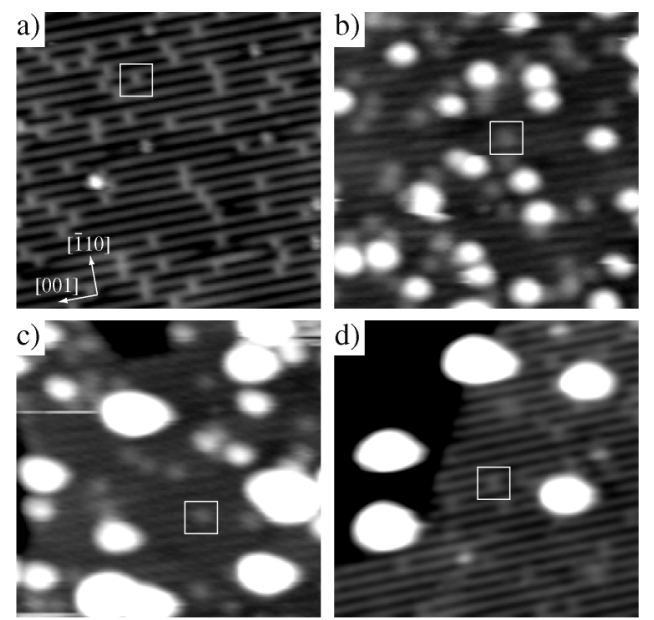

FIG. 1. (a) Clean $\mathrm{TiO}_{2}(110)$ surface with bridging oxygen vacancies. (b) -(d) $\mathrm{TiO}_{2}(110)$ surface after $4 \% \mathrm{ML} \mathrm{Au}$ deposition at 130,210 , and $300 \mathrm{~K}$, respectively. Vacancies are indicated by squares. All images are $150 \times 150 \AA^{2}$.

avoid water contamination, and we can therefore safely assign the bright spots between Ti rows to single oxygen vacancies.

After Au deposition, we observe Au clusters on the surface [Figs. 1(b)-1(d)]. The number of Au clusters increases with the amount of Au deposited. The size of the clusters depends on the deposition temperature. At the lowest temperature $(130 \mathrm{~K})$, most of the clusters contain only a few atoms and are homogeneously distributed on the terraces. High-resolution STM images reveal that the smallest protrusions found (Fig. 2) are located on the bridging oxygen rows. We assign these to single Au atoms adsorbed in a bridging oxygen vacancy (further evidence is provided below). With increasing deposition temperature, the average cluster density decreases and the average Au cluster size increases accordingly. At $210 \mathrm{~K}$, the clusters are still distributed homogeneously on the terraces but contain on average 10 atoms at a coverage of $4 \% \mathrm{ML}$ of Au. However, at $300 \mathrm{~K}$ (RT) STM images show that clusters are preferentially found at step edges and contain $\sim 30$ atoms at the same coverage.
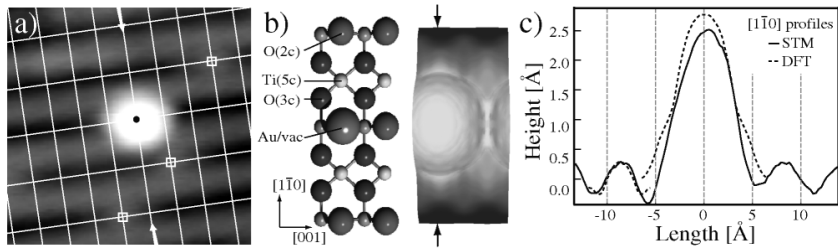

FIG. 2. (a) A closeup STM image of one of the smallest clusters observed. Vacancies are marked with squares. (b) Simulated STM image of a single Au atom trapped in an oxygen vacancy. A model of the $(2 \times 2)$ surface unit cell used in the calculations is included. (c) Line profiles comparing theory and experiment [see arrows in (a) and (b)].
To quantify the interaction of Au clusters with bridging oxygen vacancies, we have counted in high-resolution STM images the number of vacancies as well as the number of clusters before and after Au deposition at the different deposition temperatures (Fig. 3). When $\mathrm{Au}$ is deposited, we find that at all investigated temperatures, the density of oxygen vacancies decreases. This decrease is clearly not due to a "shadowing" effect from the $\mathrm{Au}$ clusters since they occupy $<15 \%$ of the surface area and thus cannot account for the observed decrease in number of vacancies. At the lowest temperature, we find close to a 1:1 correlation between the increase in number of $\mathrm{Au}$ clusters and the decrease in the number of oxygen vacancies. At higher temperatures, the decrease in the number of oxygen vacancies is considerably larger, indicating that the clusters trap several oxygen vacancies beneath them. This shows that the clusters bind at oxygen vacancy sites at all investigated temperatures.

In Table I we have further quantified and summarized the experimental findings of Fig. 3 and listed the number of $\mathrm{Au}$ atoms per cluster, the vacancy/cluster ratio, and finally the number of Au atoms per vacancy for different sets of deposition temperature and Au coverage. It should be stressed here that the numbers are averaged over many STM images. We notice from Table I that the number of oxygen vacancies trapped under each Au cluster increases with deposition temperature for a fixed coverage, but most importantly, we find that the number of $\mathrm{Au}$ atoms per oxygen vacancy is roughly constant and equal to $3-5$ in all cases.

DFT has been used to determine the binding site of $\mathrm{Au}$ on the stoichiometric and reduced surfaces. We find that

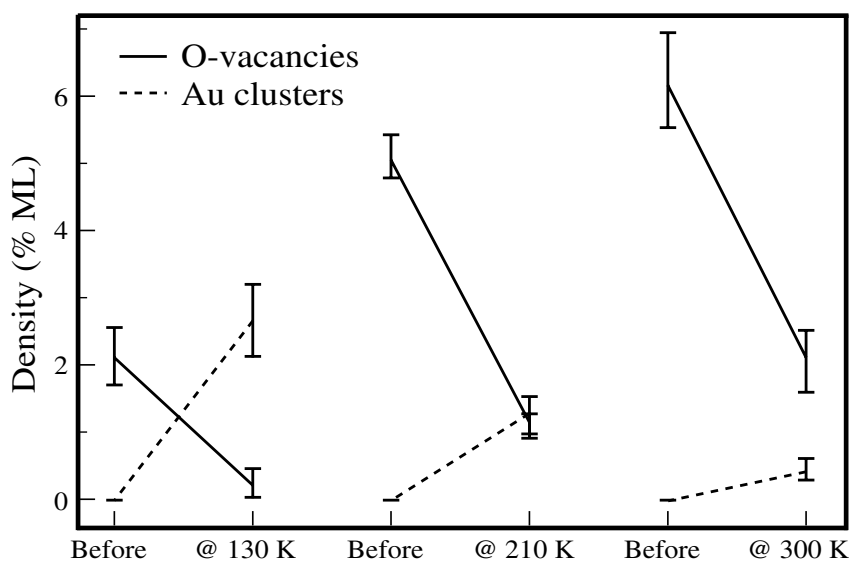

FIG. 3. Densities of vacancies and $\mathrm{Au}$ clusters before and after deposition of $\sim 0.04 \mathrm{ML} \mathrm{Au}$ at different temperatures. $1 \mathrm{ML}=1$ vacancy or cluster $/ \mathrm{TiO}_{2}(110)$ unit cell $=5.13 \times$ $10^{14} \mathrm{~cm}^{-2}$. The difference in initial vacancy concentrations at different temperatures can be explained by different sample cooling rates during preparation, since accumulation of vacancies at the surface occurs only in a narrow temperature interval $(\sim 500 \mathrm{~K}<T<700 \mathrm{~K})$ where vacancies are created, but bulksurface diffusion is hindered [13]. 
TABLE I. Interaction between oxygen vacancies and Au. Averages for different growth temperatures and coverages.

\begin{tabular}{ccccc}
\hline \hline Coverage & Temp. (K) & Atoms/clust. & Vac./clust. & Atoms/vac. \\
\hline 0.005 & 125 & $1-3$ & 1 & $1-3$ \\
0.005 & 300 & 7 & 2 & 3 \\
0.02 & 151 & 5 & 1 & 5 \\
0.04 & 130 & 5 & 1 & 5 \\
0.04 & 210 & 9 & 3 & 3 \\
0.04 & 300 & 28 & 9 & 3 \\
\hline \hline
\end{tabular}

$\mathrm{Au}$ is very weakly bound to the stoichiometric surface. A single $\mathrm{Au}$ atom binds by $1.55 \mathrm{eV}$ to the stoichiometric surface, but adsorption of a full Au monolayer (as a model of a larger cluster) is almost a thermoneutral process [10] [this fact is consistent with the difficulties arising when imaging the $\mathrm{Au} / \mathrm{TiO}_{2}(110)$ system by STM as previously stated]. The $\mathrm{Au}-\mathrm{Au}$ interactions thus dominate for larger clusters and make the $\mathrm{Au}-\mathrm{TiO}_{2}$ adhesion extremely small.

For a single $\mathrm{Au}$ atom, the most stable configuration is found to be adsorption in an oxygen vacancy site. This configuration is more stable by $0.45 \mathrm{eV}$ than Au adsorbed on the most favorable site at the stoichiometric surface (on top a bridging oxygen atom), and by $0.80 \mathrm{eV}$ with respect to adsorption on top of a fivefold coordinated $\mathrm{Ti}$ atom. The Au bond on the stoichiometric surface originates from bond polarization while the Au-vacancy bond is covalent with a very small (if any) charge transfer. This is different from $\mathrm{Au}$ adsorption in a vacancy on $\mathrm{MgO}$ where significant charge transfer occurs [7].

The smallest protrusions observed experimentally are reproduced only in simulated STM images when a single $\mathrm{Au}$ atom substitutes the missing bridging oxygen atom [see Fig. 2(b)]. These findings confirm the interpretation that the smallest protrusions centered on the bridging oxygen rows are single $\mathrm{Au}$ atoms trapped at bridging oxygen vacancies.

For low coverages we show the attachment energy of a cluster adsorbed at a vacancy, i.e., the energy gained by increasing the number of Au atoms by one (inset in Fig. 4). We find a relative instability of a cluster containing two $\mathrm{Au}$ atoms compared to a single atom adsorbed on the oxygen vacancy and a diffusing $\mathrm{Au}$ atom (on the $\mathrm{Ti}$ [001] row). In addition, $\mathrm{Au}_{3}$ is more stable than an adsorbed dimer, indicating relatively higher stability of the trimer towards decomposition. Moreover, $\mathrm{Au}_{4}$ has an electronic configuration similar to $\mathrm{Au}_{2}$ [14] and we expect it to bind weakly to a vacancy.

For large clusters, we have estimated the number of gold atoms stabilized per vacancy through a simple model. We compare the stability of a monolayer cluster (represented by a disk attached to a varying number of vacancies) to the stability of a 3D Au cluster on the stoichiometric surface (represented by a sphere) [15].

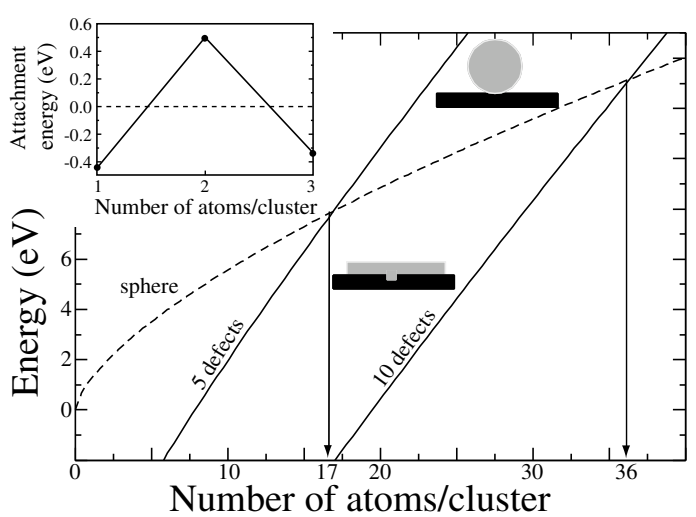

FIG. 4. Transition between adsorption on vacancies and diffusion. Solid lines show the energy for the Au disk adsorbed on a given number of oxygen vacancies (indicated in the graph). The dashed line shows the limit for diffusion, and vertical arrows indicate the actual number of $\mathrm{Au}$ atoms in the cluster at which the transition occurs. The inset shows the attachment energy for $\mathrm{Au}_{n}(n=1-3)$ adsorption on $\mathrm{TiO}_{2}(110)$.

This continuumlike model is a rough estimate and is reasonable only for fairly large clusters. The results are summarized in Fig. 4. If there are no vacancies attached to the Au cluster, a 3D cluster is always the more stable configuration but, if vacancies are included, a 2D island up to a certain size will be more stable than a 3D cluster. The maximum number of Au atoms stabilized by a group of vacancies is given by the intersection of the curves representing the two configurations. With a large number of vacancies under the deposit, such as 5 or 10, the maximum number of $\mathrm{Au}$ atoms per vacancy that can be stabilized and adsorbed in a disklike structure is about 3 . This correlates very well with the experimental observations in Table I.

To investigate the mechanism of nucleation and growth of $\mathrm{Au}$ clusters in light of the strong Au-vacancy interaction presented above, we plot in Fig. 5 the size distributions of $\mathrm{Au}$ clusters grown at different temperatures. At low temperature, the growth results in many small clusters, as also seen in Fig. 1. Upon heating, or depositing $\mathrm{Au}$ at RT, we observe fewer but larger clusters and the cluster size distribution becomes bimodal. The smallest $\mathrm{Au}$ related protrusions, i.e., single Au atom-vacancy complexes, can also be observed after deposition up to $300 \mathrm{~K}$, although their relative number decreases with increasing temperatures. When analyzing sequences of STM images we find no evidence for diffusion of single Au atom-vacancy complexes at any investigated temperature. We thus interpret the bimodal size distribution together with the stability of the smallest clusters as a sign of cluster growth through diffusion of clusters above a certain critical size [16]. Clusters nucleate primarily at oxygen vacancy sites, but upon further growth, a single oxygen vacancy can no longer stabilize the cluster, and the cluster-vacancy complex will then diffuse. While 


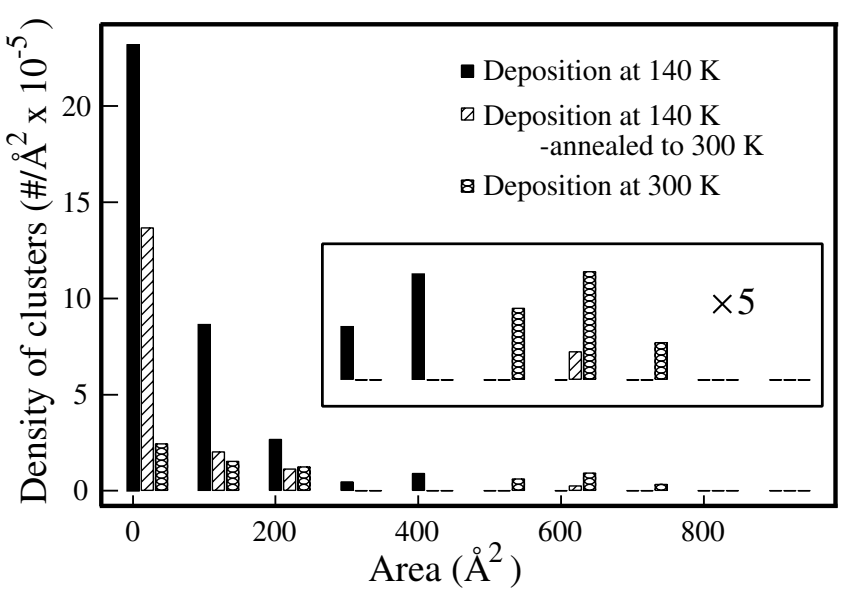

FIG. 5. Histogram of size distributions of $\mathrm{Au}$ clusters on $\mathrm{TiO}_{2}(110)$ for different deposition temperatures and annealing. All counts compare the size distribution after 0.02 ML Au. All areas were measured at a threshold level of $1 \AA$ above the medium position of the (110) plane on which the clusters were positioned.

diffusing, it can encounter either another cluster (coalescence) or other surface defects. In the former case, a larger cluster is formed that may continue to diffuse as well. If the cluster-vacancy complex encounters other oxygen vacancies it can bind to them and will therefore stabilize on the surface. It is not surprising that larger clusters formed after growth at, or annealing to, RT are found at step edges since the step edges can be considered as a collection of oxygen vacancies.

The bimodal growth model differs from other models proposed to explain $\mathrm{Au}$ growth on $\mathrm{TiO}_{2}(110)[17,18]$. Parker et al. [18] have shown that the onset of 3D growth corresponds to a coverage of $0.086 \mathrm{ML}$ on the stoichiometric $\mathrm{TiO}_{2}(110)$ surface and $0.15 \mathrm{ML}$ on the thermally reduced surface. [1 ML is defined as the packing density of an $\mathrm{Au}(111)$ plane, $1.39 \times 10^{15}$ atoms $\cdot \mathrm{cm}^{-2}$ ]. Based on the present findings, we assume that the 0.064 ML change in transition coverage originates from the presence of oxygen vacancies, and considering a typical vacancy density of $6 \%-7 \%$ ML (1 ML is here one vacancy/unit cell), we calculate that each vacancy binds $\sim 3$ atoms, the same number as deduced from Table I.

The critical cluster size for this growth mode can be deduced from the bimodal size distribution at RT in Fig. 5. We observe a minimum in the cluster population starting at $250 \AA^{2}$, i.e., clusters containing 5 to 7 atoms. Conversely, clusters containing 3 to 5 atoms are stable. This value correlates nicely with the number of $\mathrm{Au}$ atoms found per vacancy (see Table I).

In summary, we show through a combined STM and DFT study that bridging oxygen vacancies are the active nucleation sites for $\mathrm{Au}$ clusters on the rutile $\mathrm{TiO}_{2}(110)$ surface at all investigated temperatures. A single $\mathrm{Au}$ atom-vacancy complex is stable up to room temperature and a single oxygen vacancy can bind $3 \mathrm{Au}$ atoms on average. For larger clusters, the Au-substrate interface contains a high density of oxygen vacancies, which enhances the binding of Au particles to the substrate. These pieces of information may be key elements in explaining why Au nanoparticles are catalytically active for lowtemperature $\mathrm{CO}$ oxidation on the $\mathrm{TiO}_{2}(110)$ surface $[3,4]$. A new growth model involving coalescence of diffusing Au-vacancy complexes is shown to explain previous and present observations of the $\mathrm{Au} / \mathrm{TiO}_{2}(110)$ system.

N. L. thanks the Ministerio de Ciencia y Tecnología for financing through the Ramón y Cajal program. R. S. acknowledges support from the Swiss National Science Foundation. C. A. acknowledges the INFM section F through PAISS "Fast STM."

*Permanent address: Department Química Física, Universidad de Barcelona, c/Marti i Franques 1, 08028 Barcelona, Spain.

${ }^{\dagger}$ Permanent address: Trieste University and TASC-INFM Laboratory, I-34100 Trieste, Italy.

Corresponding author.

Electronic address: fbe@phys.au.dk

[1] A. Fujishima and K. Honda, Nature (London) 238, 37 (1972).

[2] A. L. Linsebigler, G. Lu, and J. T. Yates, Jr., Chem. Rev. 95, 735 (1995).

[3] M. Haruta et al., J. Catal. 115, 301 (1989).

[4] M. Valden, X. Lai, and D.W. Goodman, Science 281, 1647 (1998).

[5] C. R. Henry, Surf. Sci. Rep. 31, 235 (1998).

[6] M. Bäumer and H. J. Freund, Prog. Surf. Sci. 61, 127 (1999).

[7] A. Sanchez et al., J. Phys. Chem. A 103, 9573 (1999).

[8] R. Schaub et al., Phys. Rev. Lett. 87, 266104 (2001).

[9] J. Jacobsen et al., Phys. Rev. Lett. 75, 489 (1995).

[10] N. Lopez and J. K. Nørskov, Surf. Sci. 515, 175 (2002).

[11] U. Diebold et al., Phys. Rev. Lett. 77, 1322 (1996).

[12] U. Diebold et al., Surf. Sci. 411, 137 (1998).

[13] M. A. Henderson, Surf. Sci. 419, 174 (1999).

[14] H. Häkkinen and U. Landman, Phys. Rev. B 62, R2287 (2000).

[15] Energies are disk $E_{\text {disk }}=E_{d} n_{d}+\gamma_{0} N 2+E_{\text {edge }} \pi N^{(1 / 2)}$ and sphere $E_{\text {sphere }}=4 \gamma_{0} N^{(2 / 3)} . E_{d}$ is the $\mathrm{Au}$ binding energy to a vacancy $(=-2 \mathrm{eV}) ; n_{d}$ is the number of vacancies; $\gamma_{0}$ is the surface energy ( $=0.3 \mathrm{eV} /$ atom $) ; N$ is the number of $\mathrm{Au}$ atoms; $E_{\text {edge }}$ is the edge energy $(=0.6 \mathrm{eV} /$ atom $)$.

[16] J. Carrey et al., Phys. Rev. Lett. 86, 4600 (2001).

[17] F. Cosandey and T. E. Madey, Surf. Rev. Lett. 8, 73 (2001).

[18] S. C. Parker et al., Surf. Sci. 441, 10 (1999). 